\title{
DAS HEUTIGE EMS-ESTUARIUM MIT DEM DOLLART UND DEM UMGEBENDEN WATTGEBIET
}

\author{
J. H. VAN VOORTHUYSEN \\ (mit einer Karte, die Lage der Stationen und Profile angebend, sich als Beilage hinten im Buch \\ befindend, und einer Zahlentafel)
}

\section{EINLEITUNG}

Im Sommer des Jahres 1953, 1954 und 1956 wurden mit dem von dem Ministerium für Wasserbauverwaltung zur Verfügung gestellten Forschungsschiff „Ons Genoegen“, insgesamt 8 Wochen hydrographische, chemische und biologische Untersuchungen gemacht auf dem Wasser und auf dem Land.

Von der Nehrungsinsel Rottumeroog bis Papenburg an der Ems wurden 352 Bodenproben und etwa 160 Wasserproben gesammelt. Weiter wurden viele hydrographische Messungen durchgeführt.

Die Bodenproben wurden untersucht auf Diatomeen, Mollusken, Bryozoen, Ostracoden, Amphipoden, Copepoden, Foraminiferen und noch einige andere wirbellose Tiere. Weiter wurden von den meisten Bodenproben granulometrische Analysen gemacht. Die Wasserproben wurden biologisch und z.T. chemisch untersucht.

Die Bodenproben unter Wasser wurden mit einem Van Veen's Bodengreifer gesammelt. Für die benthonische Mikroflora und Fauna wurden nur die obersten 3 Millimeter eines Quadratmeters der Oberfläche zusammengekratzt. Für die Mollusken wurde bis $30 \mathrm{~cm}$ tief gegraben.

Die für ökologische Zwecke gesammelten Sedimentproben wurden sofort mit Alkohol übergossen, um das Protoplasma zu konservieren. Die lebende und tote Fauna und Flora wurde mittels der "RoseBengal“" Protoplasmafärbung von Walton (Vgl. VAN VoorthuYsen; die Foraminiferen des Dollart-EmsEstuarium, in dieser Abhandlung) getrennt.

Die Lage der Stationen und Profile sind auf der zugehörigen Karte verzeichnet.

Das untersuchte Gebiet liegt in einem Teil des sich an den niederländischen, deutschen und dänischen Küsten erstreckenden Wattgebietes der Nordsee, die zu einer untiefen Schelfsee gerechnet werden muß.
Nach der Einteilung der marinen Lebensräume von Remane (1940) und Hedgpeth (1957) gehört das Wattgebiet zur supra- und eu-litoralen (Eng.: intertidal) Zone. Die bis $20 \mathrm{~m}$ tiefen Rinnen könnte man vielleicht zur sub-litoralen Zone rechnen.

Die verschiedenen Autoren verfahren nicht nach denselben Salinitätsschemata der Brackwassergebiete. Die Hydrographen und Hydrobiologen benutzen das Chlorinitätsschema nach REDEKE (1933) und REMane (1940), die Stratigraphen die Formel nach VäLIKaNGAS (1933) für den Gesamtsalzgehalt oder die den fossielen Lebensräumen angepaßte Einteilung nach Hilterman (1949, vgl. untenstehende Zahlentafel).

Nach Emery und Stevenson (1957) ist ein Estuarium die breite Mündung eines Flusses oder Meeresarmes, wo die Tide mit den Flußströmungen zusammenstößt. Physiographisch sind nach beiden obengenannten Autoren Estuarien Wasserkörper, umsäumt und zum Teil vom Ozean abgeschnitten durch Landmassen, die ursprünglich durch nichtmarine Wirkungen entstanden waren. Sie stehen gewöhnlich senkrecht auf der Küstenlinie und die meisten benutzen die ertrunkenen Mündungen von Flußtälern; deshalb sind sie meistens als Beweis für Untertauchung betrachtet worden.

Frühere Untersuchungen haben es wahrscheinlich gemacht, daß die Sedimente des Wattenmeeres von der Nordsee herstammen und von den Gezeitenströmungen eingeschwemmt worden sind (СRомmelin, 1943, Postma, 1954). Das berechtigt die kürzlich von GripP (1956) gegebene Definition: „Ein Watt ist ein vom Meere in Buchten zusammengeschwemmter Sedimentkörper, dessen Oberfläche durch das von den Gezeiten bewegte Wasser annähernd in Höhe des Gezeiten-Mittelwassers gehalten wird“. Die Watten werden aufgebaut vom Meere her und sind nicht an 


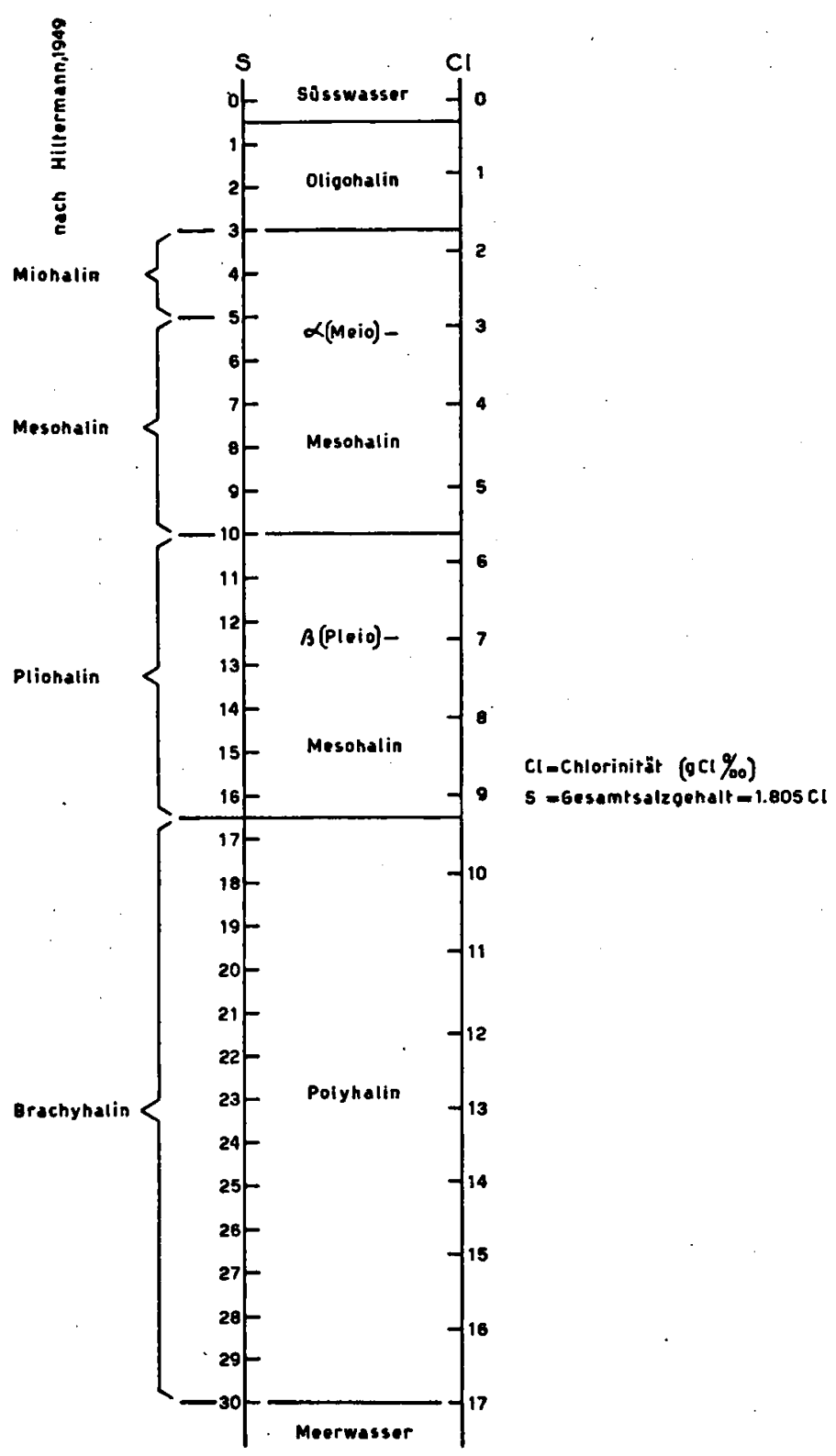

die Mündung eines Stromes gebunden.

Neuerdings haben Van Straaten und Kuenen (1957) den Mechanismus der Einschwemmung des feinen Materials näher geklärt.

Das untersuchte Ems-Estuarium mit dem Dollart und dem angrenzenden Wattgebiet ist ein gutes Beispiel für das Axiom, daß es keine exakten Grenzen in der Natur gibt. Nach Emery und Stevenson (1957) ist die charakteristischste Eigenschaft einer estuaren Umwelt die plötzliche Anderung der ökologischen Verhältnisse, die außerdem einer starken Abwechse- lung aușgesetzt sind. Die größte, auffälligste Anderung der ökologischen Verhältnisse fängt im hiesigen Untersuchungsgebiet nicht in der Nähe der Hondplate an, wo ökologisch die Ausmündung des Ems-Estuarium ins Wattenmeer angenommen werden muß, sondern gerade hinter den Nehrungsinseln Rottumeroog und Borkum. Das ist also im Widerspruch mit der genannten Auffassung von Estuarium. Man kann aber von anderen Gesichtspunkten aus das Estuarium auch nicht in der Nähe der Nehrungsinseln anfangen lassen, weil das der Definition widersprechen würde, 
daß ein Estuarium durch nicht-marine Wirkungen gebildet wird. Das Ems-Estuarium kreuzt doch ein Wattgebiet und ein Watt wird gerade vom Meere her aufgebaut. Theoretisch muß nach EMERY ein Estuarium sich selbst durch die Bildung eines Schuttkegels oder Deltas am inneren Ende verkürzen, während zu gleicher Zeit die Wellen die Küsten in der Nähe der Mündung angreifen. Erstens besitzen die meisten europäischen Estuarien aber kein Delta wegen der starken Gezeitenströmungen und des sehr feinkörnigen
Flußsediment und zweitens kann im Ems-Estuarium auch fast keinen Abrasion stattfinden infolge der schützenden Wirkung des Wattkörpers und der Nehrungsinseln.

Die landeinwärts gelegene Grenze des Estuarium kann leichter angegeben werden. Sie liegt dort, wo der Einfluß des Meeres in der Fauna und Flora nicht mehr zu beobachten ist. Sie liegt etwa $10 \mathrm{~km}$ stromaufwärts von Pogum an der Ems.

\section{SCHRIFTTUM}

Crommelin, R. D. (1943) - De herkomst van het waddenslib met korrel-grootte boven 10 micron, Verh. Geol. Mijnb. Gen., Geol. Serie, deel 13, S. 299-331.

Emery, K. O. und Stevenson, R. E. (1957) - Estuaries and Lagoons, I. Physical and Chenical Characteristics, Geol. Soc. America, Mem. 67, Vol. 1, S. 673-693.

Gripp, KaRI (1956) - Das Watt; Begriff, Begrenzung und fossile Vorkommen, Senckenbergiana lethaea, Band 37, Nr. 3/4, S. 149-181.

Hedgpeth, Joel, W. (1957) - Classification of Marine Environments, Geol. Soc. America, Mem. 67, Vol. 1, S. 17-28.

HiltermanN, H. (1949) - Klassifikation der natürlichen Brackwässer, Erdöl und Kohle, 2. Jahrg., Nr. 1, S. 4-8.

Kuenen, Ph. H. (1950) - Marine Geology, John Wiley \& Sons, Inc., New York, S. 97.
Postma, H. (1954) - Hydrography of the Dutch Wadden Sea, Thesis Groningen, Arch. Néerland. Zoologie, X, 4 e. livraison.

Redeke, H. C. (1933) - Uber den jetzigen Stand unsere Kenntnisse der Flora und Fauna des Brackwassers, Verh. int. Ver. theor. und angew. Limnologie, vol. 6, S. 46-61.

Remane, A. (1940) - Einführung in die zoologische Ókologie der Nord- und Ostsee. Die Tierwelt der Nord- und Ostsee, Grimpe-Wagler Lief. 34, Teil 1a, Leipzig.

Straaten, L. M. J. U. van, und Kuenen, Ph. H. (1957) Accumulation of fine grained sediments in the Dutch Wadden Sea, Geol. en Mijnb., Nw. Serie, 19e Jaarg., S. 329-354.

Välukancas, I. (1933) - Über die Ostsee als Brackwassergebiet, Verh. Int. Verein. theor. und angew. Limn., 6, I, Stuttgart. 